\title{
Parameters of the Protein Energy Landscapes of Several Light- harvesting Complexes Probed via Spectral Hole Growth Kinetics
}

Measurements.

\author{
Nicoleta Herascu ${ }^{1}$, Mehdi Najafi ${ }^{1}$, Alexey Amunts ${ }^{2}$, Jörg Pieper ${ }^{3,+}$, Klaus-Dieter \\ Irrgang $^{4}$, Rafael Picorel ${ }^{5}$, Michael Seibert ${ }^{6}$ and Valter Zazubovich ${ }^{1, *}$ \\ ${ }^{1}$ Department of Physics, Concordia University, 7141 Sherbrooke Str. West, \\ Montreal, Quebec, H4B 1 R6 Canada; ${ }^{2}$ MRC Laboratory of Molecular Biology, \\ Cambridge, UK; ${ }^{3}$ Technical University of Berlin, Germany; ${ }^{4}$ University of Applied \\ Sciences, Berlin, Germany; ${ }^{5}$ Estacion Experimental Aula Dei (CSIC), Avda. \\ Montañana 1005, 50059 Zaragoza, Spain; ${ }^{6}$ NREL, 1617 Cole Boulevard, Golden, \\ Colorado, USA.
}

\begin{abstract}
The parameters of barrier distributions on the protein energy landscape in the excited electronic state of the pigment / protein system have been determined by means of spectral hole burning for the lowest-energy pigments of CP43 core antenna complex and CP29 minor antenna complex of spinach Photosystem II, as well as of trimeric and monomeric LHCII complexes transiently associated with pea Photosystem I pool. It has been demonstrated that all of these complexes exhibit sixty to several hundred times lower SHB yields as compared to molecular glassy solids previously probed by means of the hole growth kinetics (HGK) measurements. Thus, the entities (groups of atoms) which participate in conformational changes in protein appear to be significantly larger and heavier than those in molecular glasses. No evidence for small $\left(<1 \mathrm{~cm}^{-1}\right)$ spectral shift tier of the spectral diffusion dynamics has been observed. Thus, our data most likely reflects the true barrier distributions of the intact protein and not

\footnotetext{
* corresponding author, vzazubov@alcor.concordia.ca

${ }^{+}$current address: Institute of Physics, University of Tartu, 142 Riia Street, Tartu 51014 Estonia.
} 
those related to the interface or surrounding host. Possible assignments of low-energy states of CP29 and LHCII are discussed in the light of the above results.

* corresponding author, vzazubov@alcor.concordia.ca ${ }^{+}$current address: Institute of Physics, University of Tartu, 142 Riia Street, Tartu 51014 Estonia. 


\section{Introduction}

Spectral hole burning (SHB) is a well-established technique which since the end of the 80 -ies ${ }^{1,2}$ has been widely and persistently employed in the studies of pigmentprotein photosynthetic complexes (PS complexes). SHB has been applied to explore electronic level structure, electron-phonon coupling and energy and charge transfer processes in a broad variety of complexes ${ }^{3-20}$, as summarized in a recent review ${ }^{21}$. In terms of low-temperature protein dynamics, the presence of low-energy excitations responsible for the $\sim T^{1.3}$ dependence of the homogeneous line width, which is characteristic for amorphous solids, was demonstrated for many PS complexes ${ }^{3-10,12}$. Broadening of the spectral holes in time has been explored for several complexes, namely the reaction centers and CP47 complexes of plant Photosystem II (PS II) and bacterial B820 dimers ${ }^{6-9}$. Later, single photosynthetic complex spectroscopy (SPCS) has become the technique of choice to study low-temperature protein dynamics in PS complexes ${ }^{22-26}$; see ${ }^{27}$ for recent review. SPCS allows exploring PS complexes one by one, allowing for great insight in the details of their individual properties and behavior, while SHB probes ensemble averages.

Nevertheless, waiting and aging time SHB experiments still continued to be employed to study low-temperature dynamics of other proteins ${ }^{28-30}$. Both SHB and single molecule spectroscopy have been applied in the research on low-temperature dynamics and energy barrier distributions of glasses and polymers ${ }^{31-37}$. In particular, hole growth kinetics (HGK) measurements have been employed in ${ }^{13,34-37}$. However, until recently the simulations of the hole burning in PS complexes (e.g. ${ }^{15}$ ) did not take into account the 
dispersive character of hole burning ${ }^{13,34-37}$ which is due to the distribution of energy barriers in the excited state of pigment / protein system.

In the last couple of years we initiated some steps towards bridging the gap between applications of SHB and SPCS to PS complexes ${ }^{38.39}$. Our analysis of the spectral hole shape evolution for the B800 band of the LH2 antenna complex of the bacterium Rhodopseudomonas açidophila involved combining parameters derived from SPCS data ${ }^{23,25,26}$ (line shift distributions, rates, photon budgets) and theoretical SHB models originally developed for glasses, which share many important properties with the proteins. The key feature of these models is the concept of two-level systems (TLS) originally introduced in ${ }^{40,41}$ to explain anomalous low-temperature heat capacity and heat conductivity in amorphous solids. In case of SHB in amorphous solids it is assumed that the barriers between two conformations of the TLS are higher in the ground state than they are in the excited electronic state of the chromophore / host system, and that the transition between the conformations, resulting in a spectral shift, occurs due to the tunneling in the excited state ${ }^{42}$. Optical spectra of amorphous systems, including proteins, are subject to non-photochemical hole burning (NPHB) ${ }^{43}$, meaning that spectral holes appear due to the rearrangement of the local environment of the pigment, not due to a photochemical reaction involving the pigment. This is a valuable feature from the viewpoint of energy transfer research in PS complexes, since the network of interacting pigment molecules remains intact. In other words, within these models SHB essentially is treated as a manifestation of light-induced spectral diffusion. Earlier SHB models developed for glasses ${ }^{13,34,35}$ took into account the excited state barrier distributions but not the anti-hole associated with the post-burn conformation. More recently, the anti-hole 
absorption has been incorporated into the model ${ }^{36}$. The latest versions of the model included also light- and temperature-induced hole filling ${ }^{37}$, but were still limited to chromophore interacting with just one TLS.

By now it is well established that standard TLS model or its further developments are not fully capable of explaining various phenomena related to spectral diffusion in proteins, both light-induced and occurring in the dark (see ${ }^{27,28}$ for recent reviews). Both SHB experiments with hole broadening over time at different temperatures ${ }^{6-9,28-30}$ (probing the dynamics of the ground electronic state of the protein-chromophore system) and SPCS experiments ${ }^{22-26}$, which, we believe, at least at liquid helium temperatures, probe the barrier distributions in the excited state of the pigment- protein system (the latter conclusion is still a matter of debate, though, ${ }^{24,38}$ ), can be interpreted with system switching between significantly more than two conformational states. Such situation is best described employing the concept of the multi-well energy landscape with several hierarchal tiers ${ }^{22,44,45}$. The one-TLS-per-chromophore approximation appears to be too crude for a protein exhibiting multi-well energy landscape, at least for the smaller-barrier landscape tiers ${ }^{22}$. Thus, in ${ }^{38}$ we introduced a SHB model allowing chromophore / protein environment system to assume multiple conformations. Interestingly, our SHB data on LH2 proved incompatible with the lowest-barrier tier SPCS dynamics reported in 22,23,25,26, while agreeing both qualitatively and quantitatively with the higher tiers dynamics. The white light-induced hole filling was not accompanied by noticeable hole broadening (i.e. no thermally-induced spectral diffusion on the smallest-shift $\left(\sim 1 \mathrm{~cm}^{-1}\right)$ tier of the energy landscape was observed on a time scale of $\sim$ hour) and it was impossible to model the holes utilizing narrow (several $\mathrm{cm}^{-1}$ ) anti-hole function and the 
hole burning yield following from the SPCS experiment parameters ${ }^{23,25,26}$ (i.e. the lightinduced spectral diffusion corresponding to that landscape tier was, if present at all, much less pronounced than in the SPCS experiments). Hole filling in the dark was negligible during the first couple of hours after burning, which indicates that the spectral diffusion on the higher-barrier tier of the energy landscape observed in SPCS experiments is also predominantly light-induced, not thermally induced. The number of possible conformations on the higher tier(s) of the protein energy landscape, however, appears to be limited. The same conclusion can be reached based on room-temperature SPCS data ${ }^{46-48}$. These arguments suggest that original SHB models, extended to include Multi-Level Systems (MLS) still may be satisfactory for these tiers. Important implication of the results briefly described above and presented in detail in Ref. 38 is that SHB, probing ensemble averages, could be used to verify if light-induced spectral diffusion behavior observed in SPCS experiments represents the behavior which is typical for the protein under study, and independent of sample preparation details (e.g. nature of the amorphous host surrounding the protein), and/or other experimental procedures. In other words, the property widely considered to be a deficiency of the SHB with respect to SPCS may be treated as beneficial, since it provides independent benchmarks for SPCS results.

As mentioned above, according to the results of Ref. 38 the spectral diffusion observed in SPCS experiments is most likely light-induced and therefore essentially the same phenomenon as NPHB. With that in mind, we are going to apply the NPHB model of Ref. 38 to determine the parameters of the barrier distributions, affecting the evolution of spectral holes during burning, for several protein antenna complexes including LHCII peripheral antenna complex, CP29 minor antenna complex and CP43 core antenna 
complex of PS II. First, it is natural to test if barrier parameters observed for LH2 in ${ }^{38}$ are typical for PS complexes in general, or are a consequence of some specific properties of LH2. Second, to the best of our knowledge no low-temperature single complex data has ever been presented for CP43 and CP29 complexes. While some low-temperature singlecomplex data is available for LHCII ${ }^{50,51,60,61}$, these papers do not focus on spectral diffusion or barrier distribution parameters. Once respective single complex data becomes available, it could be verified against our SHB-based parameters. The parameters obtained in the course of this study will also be utilized for development and refinement of light-induced spectral diffusion models simultaneously explaining both SHB and low-temperature SPCS results. Another possible application of barrier distribution parameters in photosynthetic research by means of SHB involves disentangling the effects of barrier distributions from the effects related to the distributions of the excitation energy transfer (EET) rates. When distributions of the EET rates becomes measurable in the SHB experiments on higher-energy states of the complexes, they can be compared to those theoretically predicted from the structural data, and additional constraints for transition energies of the chlorophylls in the absence of inter-pigment interactions can be obtained. The latter energies are still not precisely determined for most of the chlorophylls in most of the photosynthetic complexes.

\section{Experimental.}

2.1. Sample preparation. Spectral holes and hole growth kinetics (HGK) curves were measured for the lowest-energy regions of CP43 and CP29 complexes of PS II from spinach and of monomeric and trimeric LHCII complexes (transiently associated with 
Photosystem I, PS I) from pea. CP43 and CP29 samples were isolated and purified as described in ${ }^{18}$ and ${ }^{11}$, respectively. In fact the CP43 and CP29 samples used in this work were the same as used in ${ }^{18}$ and ${ }^{11}$, respectively; stored in the meantime at $-80^{\circ} \mathrm{C}$. As will be demonstrated later, prolonged storage did not affect the spectral properties of CP29 significantly (slight shift of the lowest-energy state has been observed, see below). Unlike most LHCII preparations reported previously, the one employed in this study was obtained in the way of PS I purification (at Tel Aviv University). Isolation of thylakoid membranes from 12-day-old pea (Pisum sativum) was performed based on the previously described method ${ }^{49}$. All the subsequent procedures were performed in dim light at 4-6 ${ }^{\circ} \mathrm{C}$. Isolated thylakoids were adjusted to chlorophyll concentration of $3.0 \mathrm{mg} \mathrm{chl} / \mathrm{ml}$ and solubilized by the detergent n-Dodecyl-ß-D-maltoside (DM) (Glycon, Inc) to give a final concentration of $1.55 \mathrm{mg}$ detergent per mg chl. Following 15 min incubation on ice, the material was ultracentrifuged at 150,000 g for $40 \mathrm{~min}$. These conditions were found to solubilize mainly PS II, ATP synthase and $b_{6} f$ complex. The resulting pellet was suspended using a glass-Teflon homogenizer in a buffer containing $0.3 \mathrm{M}$ sucrose, 20 mM Tricine (pH 8), 2 M Betain and $1 \mathrm{mM}$ phenylmethyl-sulphonyl fluoride (PMSF) at a chlorophyll concentration of $3.0 \mathrm{mg} \mathrm{chl} / \mathrm{ml}$. The material was then solubilized with 6.0 mg DM per mg chl. Unsolubilized material was removed using ultracentrifugation at 110,000 g for $20 \mathrm{~min}$. The supernatant was applied onto $15-40 \%$ sucrose gradient. PSILHCII fraction was collected and loaded onto a Diethylaminoethyl (DEAE) cellulose column (DE-52, Wattman, Inc) $(1.5$ x $18 \mathrm{~cm})$ that was pre-equilibrated with $20 \mathrm{mM}$ Tricine-Tris ( $\mathrm{pH}$ 7.4) containing $0.2 \%$ (w/v) DM. The column was washed with $25 \mathrm{ml}$ of the same buffer, and LHCII was eluted with a $0-200 \mathrm{mM} \mathrm{NaCl}$ linear gradient $(130 \mathrm{ml}$ in 
each chamber) in $20 \mathrm{mM}$ Tricine-Tris (pH 7.4) containing $0.2 \%$ (w/v) DM. First, dark green fractions containing mainly LHCII trimer and monomer complexes were properly separated from PS I and other remainder photosynthetic complexes. Fractions containing LHCII were precipitated by adding $10 \%$ (w/v) PEG6000 (Hampton, Inc) followed by centrifugation at 10,000 g for $5 \mathrm{~min}$. The pellet was dissolved in solution containing 20 mM Tricine-Tris (pH 7.4), $0.05 \%$ (w/v) DM. For further separation between trimers and monomers, the LHCII was applied onto a 10-25\% sucrose gradient containing the same buffer, and centrifuged using the SW40 rotor (Beckman, Inc) at 37,000 rpm (24,000 g) for $30 \mathrm{~h}$. LHCII appeared in two dark green bands in the middle of the tube. The lower band contained highly purified trimer complexes, while the upper band contained highly purified monomer complexes. To avoid protein heterogeneity, only the middle section of each band was collected. The material was precipitated with $10 \%$ (w/v) PEG6000 and 50 $\mathrm{mM}$ ammonium acetate and subsequent centrifugation at 10,000 $\mathrm{g}$ for $5 \mathrm{~min}$. The pellet was dissolved in solution containing $2 \mathrm{mM}$ Tricine-Tris (pH 7), 0.05\% (w/v) DM, and adjusted to a chlorophyll concentration of $3.0 \mathrm{mg} / \mathrm{ml}$ for further experimental procedures. Concentrated samples were stored at $-80^{\circ}$, and thawed up and diluted with buffer (20 mM Bis-Tris, $\mathrm{pH}$ 6.0, 0.03\% (w/v) DM for CP43, 10mM Tricine, $\mathrm{pH}$ 7.8, 0.06\% DM for LHCII and 25mM HEPES, pH 7.6, 0.06\% DM for CP29) prior to experiment.

2.2. Spectroscopic measurements were performed in a model A240 helium cryostat (Ukrainian Academy of Sciences) at 5 K. Samples were diluted with glycerol 40:60 several minutes before the start of cooling and placed in a Eppendorf Uvette featuring orthogonal optical paths of $10 \mathrm{~mm}$ and $2 \mathrm{~mm}$. Use of the Uvette allowed absorbance (10 mm path; moderate OD) and fluorescence excitation (2 mm path, 
reflection geometry; small OD to avoid reabsorption effects) measurements with the same sample. Quality of the samples and the (low) extent of aggregation in case of LHCII were confirmed by measuring their absorption spectra with Varian Cary 5000 spectrophotometer at resolution of $0.25 \mathrm{~nm}$ as well as by measuring their fluorescence spectra using Jobin-Yvon HR640 spectrograph with Princeton Instruments Pixis CCD detector. The fluorescence spectra were corrected for the system sensitivity curve. Highresolution SHB experiments were performed with Spectra-Physics/ Sirah Matisse-DS tunable dye laser (DCM dye) pumped with 6W $532 \mathrm{~nm}$ Spectra-Physics Millennia solid state laser. The Matisse-DS is actively stabilized to $<1 \mathrm{MHz}$ bandwidth and capable of seamless $\sim 45 \mathrm{GHz}$ scans even with passive frequency stabilization. The counterdrift function of the laser control electronics, including HighFinesse WS-U30 wavemeter, allows for long-term (hours) stabilization of the laser frequency to $<30 \mathrm{MHz}$ for HGK measurements. (This precision is sufficient as homogeneous line widths in photosynthetic complexes at $5 \mathrm{~K}$ are of the order of a $\mathrm{GHz}^{10,12}$.) High-resolution spectra and kinetics curves were detected in fluorescence excitation mode with Hamamatsu PMT / photon counting module (with AELP-730 interference long-pass filter, Omega, USA, and some neutral-density and conventional long-pass filters, LOMO, Russia), positioned at $90^{\circ}$ with respect to the excitation beam. Fresnel rhomb-based broadband polarization plane rotator (ThorLabs) was used to rotate horizontally polarized light emitted by the Matisse by $90^{\circ}$ in order to achieve a situation where, given the geometry of the experiment, fluorescence from preferentially excited molecules was most effectively detected. Contributions to the measured signal of the reflected excitation light or of cuvette material fluorescence were measured using a control sample containing buffer / glycerol mixture only (no 
chromoprotein) for all filter combinations used in HGK measurements, and subtracted from the respective HGK curves before fitting. The effects of the cuvette material on light polarization have been explored as well. Laser light transmitted through the polarizer exhibited degree of polarization $\left(I_{\max }-I_{\min }\right) /\left(I_{\max }+I_{\min }\right)=95 \%$ (imperfections of laser and polarizer combined). Introduction of the Uvette reduced the degree of polarization to about $90 \%$ (after passing through two cuvette walls; obviously the light is interacting with the sample after passing just through one wall of the cuvette). Thus, the birefringence of the Uvette material was ruled negligible. Excitation intensity was stabilized by a power stabilizer (BEOC) and adjusted with neutral density filters (ThorLabs). Between the burns at different wavelengths the holes were erased by heating the samples up to $\sim 150 \mathrm{~K}$. Thus, mutual interference of different holes was excluded.

2.3. Model. According to ${ }^{13,34,35,38}$, in the absence of the energy transfer (this is the case for this manuscript focusing on the lowest-energy states of the complexes) the time dependence of the absorption spectrum affected by SHB can be described with $D(\Omega, t)=1.5 \int d \omega L(\Omega-\omega) G(\omega) \int d \lambda f(\lambda) \int d \alpha \sin \alpha \cos ^{2} \alpha e^{-P \sigma \phi(\lambda) L\left(\omega_{B}-\omega\right) t \cos ^{2} \alpha}$

Here $\omega_{B}$ is the burn frequency, $P$ is the photon flux, $t$ is the burn time and $G(\omega)$ is the site distribution function (SDF), describing the probabilities of encountering different zerophonon transition frequencies. $L\left(\omega_{B}-\omega\right)$ is the single molecule absorption profile with zero-phonon line (ZPL) at the burn frequency, $\omega_{B}$. The one-phonon profile is assumed to be half-Gaussian half-Lorentzian, with FWHM values reported in subsequent text and Table 1. Multi-phonon processes are taken into account during calculation of $L(\omega)$. $\lambda$ and 
$f(\lambda)$ are the tunneling parameter and (Gaussian) distribution thereof, with mean $\lambda_{0}$ and standard deviation $\sigma_{\lambda . .} \lambda=d \sqrt{2 m V} / \hbar$, where $d$ is the displacement along generalized coordinate, $m$ is the effective mass of entity rearranging during the conformational change, and $V$ is the barrier height. Thus, tunneling parameter distribution is a reflection of the distributions of the barrier parameters of the protein energy landscape. $\sigma$ and $\phi$ are the integrated absorption cross-section of the molecule aligned with transition dipole parallel to laser polarization and hole burning quantum yield, respectively. $\alpha$ is the angle between laser polarization and transition dipole. The NPHB yield can be defined as

$$
\phi(\lambda)=\frac{\Omega_{0} \exp (-2 \lambda)}{\Omega_{0} \exp (-2 \lambda)+\tau_{1}^{-1}}
$$

where $\tau_{1}$ is, in the absence of energy transfer, fluorescence lifetime, and $\Omega_{0}=7.6110^{12} \mathrm{~s}^{-1}$ is constant pre-factor. Changing the value of this pre-factor affects the value of $\lambda_{0}$; for the purpose of comparing our results to those obtained for glasses ${ }^{34-37}$ we utilize same prefactor value. Note that the width of the Lorentzian homogeneous ZPL contributing to $L(\omega)$ is determined not by $\tau_{1}$, but by the total dephasing time $\tau_{2} .1 / \tau_{2}=1 /\left(2 \tau_{1}\right)+1 / \tau_{2}{ }^{*}$, where $\tau_{2} *$ is pure dephasing time.. Homogeneous line width is $\Gamma_{\text {hom }}=1 / \pi c \tau_{2}$, where $\Gamma_{\text {hom }}$ is in $\mathrm{cm}^{-1}$ and $c$ is speed of light in $\mathrm{cm} / \mathrm{s}$. The hole growth kinetics (HGK) curve describes the evolution of absorption in resonance with the burning laser, i.e. at $\omega_{B}$. It has been confirmed ${ }^{38}$ that reasonably narrow distributions of electron-phonon coupling strengths (similar to those observed in single complex experiments on LH2 in ${ }^{25,26}$ ) or of oscillator strength of the state being burned (which may occur in case the state possesses excitonic character) do not contribute significantly to the dispersion of the SHB kinetics 
and these distributions have been neglected for the sake of keeping calculation time within manageable limits.

The above equations are adequately describing the hole burning process at broad range of irradiation doses (not just in the shallow hole limit) but only in case NPHB antihole is ignored. Two different approaches to including NPHB anti-hole into consideration have been proposed. In ${ }^{36}$ it was assumed that the chromophore interacts with one and only one TLS of the amorphous solid, and therefore the system has perfect spectral memory (upon light- or thermally-induced hole filling the chromophore always returns to the original pre-burn absorption frequency). Recent single complex spectroscopy results ${ }^{23,25,26}$ suggest that a protein containing chromophore can assume more than two different conformations (different wells on the protein energy landscape ${ }^{22,44,45}$ ). NPHB modeling procedure has been modified accordingly in ${ }^{38}$, and same procedure will be employed here as well. Namely, it was assumed that molecules starting at $\omega_{\text {init }}$ before burning are redistributed around $\omega_{\text {init }}$ according to a certain distribution, called anti-hole function, as a result of burning. (This distribution should agree with the distribution of line shifts observed in SPCS experiments, as was the case for LH2, except for the smallest-shift tier of the energy landscape ${ }^{38}$ ). The following sequence has been repeated in a loop: After a shallow burn, the SDF of the burnt molecules, $G(\omega) \cdot\left(1-\exp \left(-\operatorname{Pt} \sigma \phi L\left(\omega-\omega_{\mathrm{B}}\right) \cos ^{2} \alpha\right)\right)$ was convoluted with the properly normalized anti-hole function, and added to the burnt SDF $G(\omega)^{\prime} \exp \left(-P t \sigma \phi L\left(\omega-\omega_{\mathrm{B}}\right) \cos ^{2} \alpha\right)$. This results in a modified shape of the SDF $G(\omega)$, without change in its normalization; modified SDF is used at the next step of the burn sequence. (One starts with $G(\omega)$ being Gaussian.) Unlike in ${ }^{36,37}$, there was no spectral memory (i.e. it was assumed, based on SPCS results for LH2 ${ }^{23,25,26}$ and LHCII ${ }^{50,51}$, that 
the single molecule line can be found at significantly more than two frequencies) and no correlation was implied between the shifts of the absorption of a molecule in the consecutive steps. The probability of burning at each step of the sequence was still determined by the standard SHB yield formula (Eq. 2). (The SHB yield can be independently estimated from photon budgets and shift rates of the SPCS experiments ${ }^{38}$ ). The software was extensively tested with various parameters and it has been confirmed that in case the anti-hole was shifted far away to the blue from the burn wavelength (and thus multiple acts of burning per single molecule were excluded), both programs (employed in this work and that of ${ }^{36,37}$, with perfect spectral memory) yielded identical results. Due to large number of parameters in Eqs. 1 and 2, the simulations described below involved fixing most of these parameters to values independently available from the literature while we were fitting our hole growth kinetics curves for the best barrier distribution parameters. Only when it proved impossible to obtain reasonable fits using parameters previously reported, did we engage in varying other parameters, e.g. electronphonon coupling ones, and in discussing the reasons for the discrepancies.

\section{Results}

3.1. Absorption, fluorescence and SHB action spectra. The sufficiently precise determination of the SDF parameters of the state being burned is important for successfully obtaining the distribution of barriers on the protein energy landscape in the excited electronic state. Figure 1 contains the absorption spectra of complexes being explored as well as the low-dose $\left(<2 \mathrm{~mJ} / \mathrm{cm}^{2}\right)$ hole burning action spectra (hole depth dependence on the wavelength for fixed irradiation dose) for the lower-energy states of 
the complexes. Blue curves represent emission spectra obtained with $650 \mathrm{~nm}$ excitation. According to conventional wisdom, the HB action spectrum represents the SDF of the lowest-energy state of the complex. More precisely, in case of strong overlap between several bands, the action spectrum represents a weighted sum of the parts of the SDF of the two (or more) pigments which can be the lowest-energy ones in respective individual complexes due to disorder ${ }^{52}$. (Similar logic has been applied to LHCII before ${ }^{53}$, with identical SDF assumed for all pigments within a "cluster" containing multiple molecules.) The deconvolution of the CP43 spectra was presented in ${ }^{52}$. The details on other complexes are presented in the Supplemental Section. Briefly, the more or less pronounced asymmetry of HB action spectra, which is due to EET between pigments (or groups of pigments in case of delocalized excitonic states) with uncorrelated SDF, results in Gaussian fits to these spectra (dashed black curves in frames B-D of Figure 1) being relatively poor. In particular, these fits underestimate the red tails of both HB action and absorption spectra of the complexes. Thus, for all complexes the pigment 1 SDF ("pigment 1 " refers to the pigment which is on average the lowest-energy one in the complex; in case of trimeric LHCII "complex" refers to monomer) has been chosen in such way as to correctly approximate the low-energy tail of the HB action and absorption spectra. This resulted in the lowest-states SDF being systematically broader than previously reported based on Gaussian fits to HB action spectra ${ }^{10,17,19}$. The pigment 2 and if necessary pigment 3 SDF have then been chosen to fit the remaining absorption. It is important to remember that the exact values of peak and width of the lowest-state SDF do not significantly affect the HGK parameters as long as burning is performed at the low-energy side of the band and the SDF provides a good fit to the absorption and HB 
action spectra at burn wavelength and at longer wavelengths. However, parameters resulting from the fit to HGK data, i.e. those of the $\lambda$-distribution, are sensitive to the oscillator strength of the state being burned. (The oscillator strengths of the states in photosynthetic complexes may differ from one chlorophyll equivalent due to excitonic interactions ${ }^{15,62}$.) Thus, the purpose of the analysis was also the determination of the realistic range of the oscillator strengths of the lowest state(s).

Within the framework outlined in Ref. 52, not only the HB action spectra, but also the non-resonantly burned spectral holes (as in ${ }^{11,17,19}$ ) and the fluorescence spectra contain two (or more) contributions. We stress that only one pigment per individual complex is the lowest-energy one and, therefore, experiences the most effective burning and contributes to the fluorescence spectrum; however due to disorder the pigment (or state) which happens to be the lowest-energy one may vary between individual complexes in the ensemble. The differences in the shape of high-dose and low-dose HB action spectra, and of non-resonantly burned holes ${ }^{17,19}$ can then be attributed to the differences in SHB efficiencies $\sigma \phi L\left(\omega_{B}\right)$ between different pigments (states) in the complex. In this study we are focusing on the lowest-energy pigments of the complexes as this guarantees that the observed dispersion of the hole growth kinetics is not contributed to by the dispersion of the excited state lifetimes (dispersion of the homogeneous line widths), and all HGK dispersion can be attributed to the distribution of the barriers between different conformational states of the protein. The parameters of the SDFs of the lowest-energy pigments are summarized in Table 1.

3.1.1. CP43. The absorption spectrum of CP43 core antenna complex of PS II is presented in Figure 1A. It very closely resembles those reported earlier ${ }^{12,14}$ and is nearly 
identical to recent data ${ }^{18}$. The hole-burning action spectrum of CP43 (also presented in Fig. 1A, diamonds) and its interpretation were discussed in details in ${ }^{18,52,54}$. Briefly, the action spectrum of CP43 has contributions from A- and B-state chlorophylls (B state seems to be somewhat delocalized), with either one or another happening to be the lowest-energy chlorophyll in a given single complex due to static site energy disorder. The A-state ${ }^{12}$, likely localized on Chl $44{ }^{54}$ (notation by Loll et al ${ }^{55}$ ), is the major contributor to absorption at $686.8 \mathrm{~nm}$ where the HGK measurement described below was performed (arrow). Note that the action spectrum normalized to the absorption spectrum in the region dominated by the A state overshoots the absorption in the region of the narrow B state at $683 \mathrm{~nm}$. This indicates that the hole burning effectiveness is couple of times larger for the B state than for the A state.

3.1.2. CP29: The absorption spectrum of CP29 (Figure 1B) is very similar to that reported in ${ }^{11,17}$. The most prominent peak is located at $674.8 \mathrm{~nm}\left(674.5\right.$ in $\left.^{11}\right)$. The Chl $b$ bands are located at $638.5 \mathrm{~nm}$ and $650.3 \mathrm{~nm}$ (638.5 and $650.0 \mathrm{~nm}$, respectively in ${ }^{11}$.) As in ${ }^{11,17}$, the low-energy absorption tail tapers off by $\sim 690 \mathrm{~nm}$. At the first glance, prolonged storage at $-80 C^{0}$ did not affect the properties of the sample. The hole burning action spectrum and fluorescence spectrum, though, exhibited somewhat larger red shift with respect to earlier-published results ${ }^{11,37}$ (although the magnitude of the action spectrum shift appears to be irradiation dose dependent, see below). Concerning the HB action spectrum, we utilized approaches, which are significantly different from those of ${ }^{11}$, where relatively intense burning $\left(0.05 \mathrm{~J} / \mathrm{cm}^{2}\right)$ and low read resolution $\left(0.5 \mathrm{~cm}^{-1}\right)$ were employed. Instead, we used the dye laser in high resolution mode and followed the hole growth kinetics for the initial stage of burning at various wavelengths. The irradiation 
dose of $0.005 \mathrm{~J} / \mathrm{cm}^{2}$, ten times smaller than in ${ }^{11}$, still resulted in fractional hole depths being larger than $25 \%$ for the lower-energy edge of the absorption band. Thus, Figure 1B reports two lower-dose action spectra - for irradiation dose of $0.0014 \mathrm{~J} / \mathrm{cm}^{2}$ (green triangles) and $0.0002 \mathrm{~J} / \mathrm{cm}^{2}$ (black diamonds). Use of low doses guarantees the holes were far from the onset of saturation. Both action spectra are normalized to fit the lowenergy region of the absorption spectrum. The lower-dose HB action spectrum is peaked at $679.7 \pm 0.5 \mathrm{~nm}$ (significantly different from $678.4 \mathrm{~nm}$ reported in ${ }^{11}$ and used in ${ }^{17}$ ) and is noticeably asymmetrical. Note, however, that the uncertainty in the determination of the peak position is quite high due to poor signal to noise ratio. The higher-dose action spectrum is peaked at $679.4 \pm 0.3 \mathrm{~nm}$ and is somewhat more symmetrical (triangles). We stress here that hole burning is effective for the red-most tail of the spectrum. (Although burning in the red edge was observed in ${ }^{11}$ and especially in ${ }^{17}$, a Gaussian was used to fit the HB action spectrum, which resulted in red edge of the actual SDF not being included into SDF used in simulations of various hole-burned spectra). Comparison between absorption and action spectra indicates that absorption of CP29 is dominated by easily burnable lowest state at wavelengths longer than approximately $682 \mathrm{~nm}$. Fluorescence spectrum of CP29, obtained with $650 \mathrm{~nm}$ excitation is also depicted for comparison (blue curve). It is peaked at about $679.6 \mathrm{~nm}$, only $0.4 \mathrm{~nm}$ to the red with respect to 679.2 reported in Ref. 17. The non-resonantly burned broad spectral hole (not depicted) is peaked at $\sim 679.5 \mathrm{~nm}$ for $15 \mathrm{~J} / \mathrm{cm}^{2}$ and at $\sim 679.3 \mathrm{~nm}$ for $300 \mathrm{~J} / \mathrm{cm}^{2}$ at $\lambda_{\mathrm{B}}=658 \mathrm{~nm}$, shifted by $1 \mathrm{~nm}$ with respect to $678.2 \mathrm{~nm}$ reported in ${ }^{11}$ for $500 \mathrm{~J} / \mathrm{cm}^{2}$. Apparently, the HB action spectrum exhibits noticeable irradiation dose dependence. Extrapolating that dependence to higher irradiation doses, such as used in ${ }^{11}$, one could expect further blue shift of the 
action spectrum and better agreement between our results and those of ${ }^{11,17}$. In other words, we believe that in reality the shift of the lowest-energy state(s) resulting from prolonged storage of the sample is small and find it appropriate to report the data obtained for this sample in a manuscript devoted to energy landscape barrier distributions. Allowing for two (or more) different contributions to the fluorescence origin (according to the analysis presented in Supplemental Section; similar to that employed in ${ }^{52}$ ) easily explains the wavelength dependence of the electron-phonon coupling reported in the FLN experiments ${ }^{17}$. With that in mind, we utilize the longerwavelength $S$ value of $0.65{ }^{17}$ as initial guess in subsequent HGK simulations for the longest burn wavelength. Comparing our results with those by Huyer et al. ${ }^{56}$, we suggest that fluorescence lifetimes for on average lowest-energy and second-lowest energy pigment, respectively, in CP29 are 4.8 and 2.6 ns, and use these numbers as $\tau_{1}$ (see Eq. 2, Section 2.3) in the simulations. The suggested lowest-state parameters are reported in Table 1.

3.1.3. LHCII. The absorption spectra of trimeric and monomeric LHCII are presented in Figure 1C and 1D. The latter is peaked at $675.9 \mathrm{~nm}$, and the former at 675.3 $\mathrm{nm}$. Other prominent peaks are located at $649.6 \mathrm{~nm}$ and $\sim 671 \mathrm{~nm}$. These values are practically identical to those reported in ${ }^{19}(676.0,671.0,649.5 \mathrm{~nm}$, respectively, for monomer, with main peak slightly blue-shifted for trimer), despite LHCII complexes explored here and in ${ }^{19}$ belonging to different organisms, pea and spinach, respectively. For aggregated spinach LHCII the main peak has been reported at $676.3 \mathrm{~nm}$ in ${ }^{57}$. To further confirm the absence of significant aggregation in our samples, the fluorescence spectra were measured at both $5 \mathrm{~K}$ and $80 \mathrm{~K}$ and compared with those from ${ }^{19,58}$. The $5 \mathrm{~K}$ 
fluorescence spectrum of the trimeric LHCII sample is depicted in Figure 1C (blue curve) along with the absorption and action spectra. Obviously, our fluorescence spectrum is similar to the spectra of well-solubilized (non-aggregated) LHCII reported earlier. No significant emission bands have been observed at $700 \mathrm{~nm}$ and longer wavelengths. The aggregation state of the LHCII is important since it affects the SDF parameters ${ }^{57}$ and, even more importantly, the lifetimes of the lowest-energy states ${ }^{58}$ that are one of the key model parameters $\left(\tau_{l}\right)$. The shoulder at $\sim 671 \mathrm{~nm}$ is not as well-resolved in the case of pea. Despite some differences, qualitatively, all the tendencies observed before for LHCII from spinach are present in the case of pea: the spectrum of the monomer is somewhat less structured, slightly red-shifted and with stronger lowest-energy region absorption.

The SHB action spectrum for LHCII trimer, Figure 1C, is clearly narrower than for monomer, it is peaked at $678.5 \mathrm{~nm}$ and has the FWHM of $90 \mathrm{~cm}^{-1}$. This could be compared to the data for spinach LHCII trimer from ${ }^{10}$, where Gaussian peaked at 678.2 $\mathrm{nm}$ and with the width of $85 \mathrm{~cm}^{-1}$ was used to fit high-dose $\mathrm{HB}$ action spectrum. Earlier work by Reddy et al. ${ }^{59}$ on samples which later were ruled partially aggregated ${ }^{57}$, reported the $\mathrm{HB}$ action spectrum peaked at $679.3 \mathrm{~nm}$ and with the width of $110 \mathrm{~cm}^{-1}$ for almost saturated ZPH. Interestingly, much larger fraction of the absorption spectrum can be fitted to the low-dose action spectrum in the case of the LHCII trimer than in the case of the monomer. Due to the somewhat asymmetric shape of the trimer action spectrum (pronounced red tailing), it is more obvious than in the case of the monomer that trimer HB action spectrum most likely contains more than one component, much like that of CP43. 
The monomer's SHB action spectrum (Figure 1D), obtained with irradiation dose of $0.0018 \mathrm{~J} / \mathrm{cm}^{2}$ is somewhat asymmetrical and is peaked at $680.2 \mathrm{~nm}$, slightly to the red compared to $679.6 \mathrm{~nm}$ recently reported for spinach monomer at slightly higher dose of $0.003 \mathrm{~J} / \mathrm{cm}^{2}{ }^{19}$. The FWHM of our low-dose action spectrum is $\sim 120 \mathrm{~cm}^{-1}$. Note that since action spectrum is somewhat asymmetrical, we are reporting the true FWHM value, not the result of a Gaussian fit. Comparing the absorption and action spectra one can notice that burning at wavelengths longer than $684 \mathrm{~nm}$ would guarantee that only the lowestenergy state is probed. Parameters of the SDF of the lowest states of the LHCII trimer and monomer used in subsequent modeling were obtained using the procedure described in ${ }^{52}$ and are presented in Table 1 (Details are presented in Supplemental Section.)

\subsection{Hole growth kinetics}

Figure 2 represents the hole growth kinetics curves for CP43 (Fig. 2A) at 686.8 nm, CP29 (Fig. 2B) at $681.7 \mathrm{~nm}$ (also probed at 680.5 and $679.0 \mathrm{~nm}$ ), LHCII trimer (Fig. 2C) at $683.1 \mathrm{~nm}$ (also probed at $682.3 \mathrm{~nm}$ ) and LHCII monomer (Fig. 2D) at $684.2 \mathrm{~nm}$ (also probed at 683.7 and $682.5 \mathrm{~nm}$ ) respectively, with fits according to Eqs.1 and 2 with modifications involving anti-hole treatment as described in Subsection 2.3. The integrated absorption cross-section of isolated Chl $a$ molecule oriented with transition dipole moment parallel to the excitation light electric field ( $\sigma$ in Eq. 1 ) was taken to be $4.5 \cdot 10^{-13} \mathrm{~cm}^{2} \mathrm{~cm}^{-1}$. This estimate is based on the Chl $a$ molar extinction coefficients in methanol and diethyl ether ${ }^{63}$. The $\mathrm{cm}^{2} \mathrm{~cm}^{-1}$ units are used here since in our software the

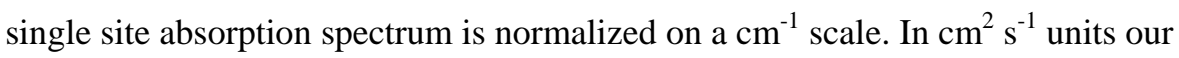
integrated cross-section estimate is equivalent to $1.410^{-2}$, which could be compared to 
$1.75 \cdot 10^{-2}$ reported for APT in hyperquenched glassy water ${ }^{37}$ or $8 \cdot 10^{-3}$ in orthodichlorobenzene glass ${ }^{36}$. Before proceeding to describe the HGK results and their fits for particular complexes, additional qualitative comments are in order. As the model employed contains many potentially adjustable parameters, one needs to develop a general understanding of how changing each of those parameters affects the theoretical HGK curves. First of all, most of the parameters were obtained from independent measurements described in the literature and were fixed during the fitting procedure. The parameters most strongly influencing the shape of the HGK curve are the mean and the width of the tunneling parameter distribution, and the Huang-Rhys factor $S$, describing the strength of the electron-phonon coupling. It is well known that $S$ can be estimated based on the maximal achievable depth of the resonant $\mathrm{ZPH}$. Thus, a rough estimate of $S$ can be obtained from the HGK curve itself even without any fitting. Other parameters affecting the maximal hole depth are the shapes of the phonon sideband and of the antihole function. The mean of the tunneling parameter distribution affects overall burning speed (i.e. if the HGK curve is shifted towards higher irradiation doses), while the width of that distribution determines the qualitative behavior of the HGK curve. Larger $\sigma_{\lambda}$ results in HGK curve (depicted on a logarithmic scale) resembling the straight line, smaller $\sigma_{\lambda}$ results in HGK curve being more sigmoidal. Thus, in our fitting procedure, we initially fixed all parameters except $\lambda_{0}$ and $\sigma_{\lambda}$, achieved the best possible fit to the experimental data, and then attempted to fine-tune the value of $S$, the shape of the phonon sideband and of anti-hole function (in this particular order) to improve the fit further. The resulting values of tunneling distribution parameters are summarized in Table 1. 
3.2.1. CP43. The shape of the SDF of the A-state of CP43 is well established 12,18,52. The $5 \mathrm{~K}$ homogeneous line width of $0.03 \mathrm{~cm}^{-1}$ was obtained from ${ }^{12}$. This width is determined mainly by pure dephasing, since radiative lifetime is as long as 3.5 ns ${ }^{64,65}$. The electron-phonon coupling parameters (Huang-Rhys factor $S$ and the parameters describing the PSB shape, which affect single-site spectrum $L(\omega)$ ) were obtained from the fits to holes from ${ }^{12,16}$ and from our old unpublished data. As demonstrated in ${ }^{14,16}$, the electron-phonon coupling for the CP43 A state is weak, $S \sim 0.25$, and the phonon sideband is peaked at $17 \mathrm{~cm}^{-1}$. These values are in agreement with extremely small Stokes shift observed for CP43 ${ }^{12,18}$. Note that within the framework of the model for EET between two quasi-degenerate states described in ${ }^{52}$, the small contribution of the B state which might still be present at $686.8 \mathrm{~nm}$ belongs to the B-type pigments being the lowestenergy pigments in the complex, and therefore, further downhill EET from these pigments is impossible. Consequently, contrary to what ${ }^{16}$ suggests, non-resonantly excited hole burning (or "photoconversion" ${ }^{14}$ ) does not contribute to the low-energy sideband in the respective hole spectrum, and the whole that sideband is a pseudo-PSB. The latter assignment is supported by the observation that the gap between the burn wavelength and the wavelength where this pseudo-PSB feature tapers off stays constant for burn wavelengths longer than $684 \mathrm{~nm}{ }^{16}$. It appears that the shape of the pseudo-PSB in case of $684<\lambda_{\mathrm{B}}<686 \mathrm{~nm}$ is determined by the actual shape of the phonon sideband, not by the shape of the SDF, and, therefore, we used the phonon sideband shape following from SHB data (See Table 1). As a result somewhat broader Lorentzian contribution to PSB width was employed here compared to ${ }^{12,18}$. There is general agreement that the Astate of CP43 is localized on a single Chl a molecule, and, therefore, the integrated 
absorption cross-section was taken to be equal to that of one Chl $a$ molecule. Finally, we used ${ }^{16}$ and our own unpublished data to estimate the shape of the anti-hole function. It is clear that the anti-hole is distributed both to the blue and to the red of the zero-phonon hole. Due to interference between the red fraction of the anti-hole and pseudo-PSB it is unclear if the anti-hole is on average blue-shifted with respect to the ZPH or not. We will use the Gaussian anti-hole function with the width of $40 \mathrm{~cm}^{-1}$, which is centered on $\omega_{\text {init }}$. Introducing slight blue shift did not affect final results significantly. As can be seen in Figure 2A, by the end of the burn, the hole reaches fractional depth of $65 \%$, which is somewhat less than $78 \%$ corresponding to $S=0.25$. Perfect fit between experimental HGK curve (black) simulation result (red) can be obtained for $S=0.3, \lambda_{0}=11.0$ and $\sigma_{\lambda}=1.0$. The remaining discrepancy between the observed fractional depth of the hole and $\exp (-S)$ is due to anti-hole influence. The insert of the Figure 2A contains hole spectrum measured at high resolution after the HGK measurement. The 65\% deep hole still has the width of 6.9 GHz only, which indicates that it is far from saturated. No significant anti-hole contributions are observed within the $45 \mathrm{GHz}$ scan range, which is in agreement with broad (30-40 $\mathrm{cm}^{-1}$ ) anti-hole function employed in our simulations.

3.2.2. CP29. According to ${ }^{11}$, the $5 \mathrm{~K}$ homogeneous width for $\mathrm{CP} 29$ is $0.04 \mathrm{~cm}^{-1}$. The electron-phonon coupling parameters were obtained from ${ }^{17}$. One should note, however, that changes in the parameters of the lowest-state SDF may result in some changes of the parameters of the electron-phonon coupling in order to produce the same delta-FLN spectra as reported in ${ }^{17} .4 .8 \mathrm{~ns}$ lifetime of the lowest state was assumed ${ }^{56}$. The measured HGK curve and the results of simulations are presented in Figure 2B for burn wavelength of $681.7 \mathrm{~nm}$. At shorter wavelengths for the same tunneling distribution 
parameters the theoretical HGK is faster than observed. According to ${ }^{17}$ the wavelength dependence of the electron-phonon coupling was observed in CP29, with $S$ decreasing towards the shorter wavelengths. Thus, one would expect experimental HGK becoming somewhat faster, not somewhat slower (as observed) towards shorter wavelengths, if the only state being burned at shorter wavelengths was the same as was burned at $681.7 \mathrm{~nm}$. The opposite tendency means that burning at 680.5 and especially $679.0 \mathrm{~nm}$ simultaneously probes two different pigments, with the shorter-energy one exhibiting lower SHB yield than the state dominating absorption at $>681.7 \mathrm{~nm}$. Fitting the HGK curves at shorter wavelengths requires using two bands for simulations rather than one. $S=0.4{ }^{17}$ was used for the second-lowest energy band. In this case reasonable (but not perfect) fit to the higher-energy HGK curves was achieved for $\lambda_{0,2} \sim 10.5$. This corresponds to $\mathrm{HB}$ rate for the second state being approximately $1 / 3$ of that for the first state, in fair agreement with estimates made above based on action spectrum shape alone $(1 / 2)$.

3.2.3. LHCII. The $5 \mathrm{~K}$ homogeneous line width was assumed to be $0.04 \mathrm{~cm}^{-1}$ for both monomer and trimer based on the results of ${ }^{10}$. This value is in reasonable agreement with the $\sim 0.1 \mathrm{~cm}^{-1}$ width of the $\sim 15 \%$ hole obtained with $\sim 2 \cdot 10^{-3} \mathrm{~J} / \mathrm{cm}^{2}$ at $682.6 \mathrm{~nm}$ for LHCII monomer in this work (see insert in Figure 2D). According to ${ }^{56}, 10$ K fluorescence decay kinetics of non-aggregated LHCII has two components, 1.8 and 4.4 ns; the latter value, corresponding to the more red-shifted component, was used in this work to simulate the lowest-energy HGK curves for both monomer and trimer. Concerning the electron-phonon coupling parameters, we utilized those from ${ }^{19}$ as initial guesses. However, due to the changes in the SDF parameters proposed in Table 1, the 
actual phonon sideband shape may differ somewhat from that suggested in ${ }^{19}$. Moreover, as can bee seen from HGK curves for both monomeric and trimeric LHCII, the maximal fractional hole depth ( $45 \%$ for monomer and $~ 35 \%$ for trimer) is significantly smaller than expected for $S=0.6$ (monomer) and $S=0.8-0.9$ (trimer) reported in ${ }^{19}$ and earlier works. The fits employing the initial guess values of $S$ were relatively poor, and resulted in $\lambda_{0}>12$ for the lowest-energy bands of both monomer and trimer. It was also clear that in order to simulate the early leveling off of the kinetics at high irradiation doses one had to introduce high values of $\sigma_{\lambda}>2.5$, which in turn resulted in poorer fit to the beginning of the curves. Therefore, we allowed $S$ to increase significantly for both monomeric and trimeric LHCII. Simultaneously, we decreased the width of the Lorentzian part of the PSB somewhat to arrive to approximately the same simulated overall shape of the phonon sideband as in $\Delta$-FLN spectra reported in ${ }^{19}$. (Alternative/modified explanation for the discrepancies will be presented in the Discussion section). One should also note that producing acceptable fits for shorter burn wavelength holes (not depicted) required that the second-lowest state (with parameter different from those for the first state) contributes significantly to absorption already at $682 \mathrm{~nm}$ (monomer) and $681 \mathrm{~nm}$ (trimer), respectively. This in turn places limitations on the oscillator strength of the lowest-energy state. The fits presented in Figures 2C and 2D were obtained assuming that the oscillator strength of the two lower-energy states of both the monomeric and trimeric LHCII does not exceed 1.2 Chl $a$ equivalents, in agreement with super-radiance data ${ }^{62}$, non-resonant SHB data from ${ }^{19}$ and this work (absence of high-energy satellite holes of significant magnitude) and with the deconvolutions reported in the Supplemental Section. Again, fit parameters are summarized in Table 1. 


\section{Discussion.}

As evident from the data presented in Table 1, the parameters of the tunneling distribution observed for photosynthetic protein complexes are significantly different from those reported for hyperquenched glassy water and simple organic glasses ${ }^{34-37}$. The LH2 antenna complex, which was explored in detail earlier ${ }^{38}$ and that contains bacteriochlorophyll $a$, not chlorophyll $a$, exhibited parameters in the same range $\left(\lambda_{0}=10.3 \pm 0.2, \sigma_{\lambda}=0.7 \pm 0.1\right)$. Note that small value of $\sigma_{\lambda}$ correlates with small inhomogeneous width of the B800 band, $\sim 130 \mathrm{~cm}^{-1}$. It also is in agreement with our results that LHCII exhibits higher energy barriers than LH2 at room temperature ${ }^{66}$. Roughly, the hole burning yield is sixty to several hundred times lower in protein complexes than in hyperquenched water and organic glasses ${ }^{34-37}$. This is reasonable, taking into account that the entities experiencing light-induced fluctuations (tunneling) are expected to be larger and heavier in protein than in the case of amorphous host comprised of small molecules. It is tempting to suggest that these entities are the whole protein sidegroups. Just like in LH2, we did not observe any evidence for fast spectral diffusion, which is likely associated with TLS of the amorphous host surrounding the pigment-protein complexes or with surface TLS ${ }^{25}$. There was no pile-up of anti-hole absorption in the immediate vicinity of the resonant hole, which would be expected if the majority of molecules were capable of experiencing small, $<1 \mathrm{~cm}^{-1}$ shifts upon burning ${ }^{23,25,26}$. As the homogeneous line widths are very small in the complexes explored in this paper compared to B800 band of LH2, the molecules experiencing spectral shifts as small as several $\mathrm{GHz}$ would become virtually unavailable for burning and would stay in the vicinity of the original resonant hole for long enough time to be detected. Summarizing, 
we believe our data reflects the true barrier distributions of the intact protein and not that of the interface or surrounding amorphous host ${ }^{25}$.

The differences between tunneling parameter distributions from complex to complex obviously reflect the differences in the protein environment of the respective chlorophyll molecules. In this respect it is instructive that these parameters are the same, within experimental errors, for the lowest-energy states of monomeric and trimeric LHCII. This indicates that the lowest-energy state of this complex most likely is contributed to by the same pigment(s) in both monomer and trimer. Interestingly, the barrier distribution of the lowest-energy state of CP29 is significantly different from that of the LHCII. This suggests that either the lowest state is localized on completely different chlorophyll(s) in CP29 and LHCII, or that the excitonic lowest state of LHCII is significantly contributed to by a chlorophyll molecule either absent in CP29, or if present, then in significantly different environment. In this respect it is worthwhile to recall the results of the recent excitonic calculations performed based on the high-resolution structure of LHCII (the structure for CP29 is not available, but similarities are expected based on genetics). Several groups ${ }^{67-69}$ reported the results of modeling, including that of not only absorption, LD and fluorescence spectra, but also of various 1D and 2D timedomain data. It has been concluded that Chl 604, proposed by Pieper et al. to be the lowest-energy pigment in the complex ${ }^{19}$, is required to absorb at much higher energy, and the lowest-energy state was attributed to Chls 610, coupled to 611 and 612. On the other hand, the non-resonant SHB experiments (Ref. 19 and this work) indicate that the lowest-energy chlorophyll in LHCII is not in appreciable excitonic interaction with other Chls $a$ of the complex. Assignment of the lowest state to either the Chl 604 or to the Chl 
610-611-612 trimer is in agreement with the rate of EET between the lowest-energy states of the adjacent monomers being negligible, as these pigments are situated far from the interface between adjacent monomers and far from identical Chls of these adjacent monomers, see Figure 3 . Although according to ${ }^{10}$ the three lowest-energy states of the LHCII trimer are the lowest-energy states of each adjacent monomer, connected by EET (with SDF peaked at 679.8, 678.4 and $677.1 \mathrm{~nm}$ respectively and having the widths of $\sim 70 \mathrm{~cm}^{-1}$ each), multiple arguments can be presented against the inter-monomer EET. Even in Ref. 10 the high-dose HB action spectrum was assumed to be the sum of the three bands listed above, not only the lowest-energy band alone, implying that energy transfer between adjacent monomers is relatively slow. On the other hand, the presence of the irradiation dose dependence of the position of the broad non-resonantly burned hole seems to indicate that SHB yield is varying between the lowest-energy states of the three monomers, suggesting some inter-monomer energy transfer is lowering the yield for two of the pigments (EET time should be used as $\tau_{l}$ in Eq. 2 in case EET is present). It has been noticed that the position of the broad non-resonant hole resulting from higherfrequency illumination followed by energy transfer is irradiation dose dependent in trimer, but not in monomer ${ }^{19}$. Our results (not depicted) indicate that such dependence, though weaker, is present also for LHCII monomer. In principle, the blue shift of the nonresonant hole may be alternatively explained by the fact that a red shift upon burning results in molecule still staying available as an energy acceptor and for subsequent burning, while the blue shift may transform the molecule into the second-lowest energy one and reduce its HB yield according to Eq.2. Stronger dependence of non-resonant hole position on dose in case of the trimer is in agreement with significantly increased $\mathrm{HB}$ 
yield for the second (or third) state (see below). Three narrow lines per single LHCII trimer, with approximately equal intensities, have been reported in the majority of the single trimer low-temperature emission spectra ${ }^{50,51,60,61}$, indicating the relative unimportance of inter-monomer EET. Several-step bleaching of single trimer fluorescence (integrated fluorescence, all wavelengths combined), accompanied with the changes in the degree of polarization, was also observed, which is consistent with the weakness of inter-monomer EET. One line and single-step bleaching was observed for single monomeric LHCII complexes ${ }^{51}$. One should note that effective inter-monomer EET should suppress the action spectrum, ultimately bringing its integral intensity down to a value corresponding to one Chl $a$ per LHCII trimer, i.e., per 42 chlorophylls (or slightly more than $1 / 42$ for lowest state being delocalized excitonic one). Even a quick look at Figures 1C and 1D allows noticing that this is not the case. Thus, the assumption of negligible inter-monomer EET appears reasonable. (We would like to clarify that in the absence of the inter-monomer EET within the LHCII trimers, the "pigment 1" SDF reported in Table 1 is a superposition of the SDFs of the on average lowest-energy pigments in three adjacent monomers, which may be either Lhcb1, Lhcb2, or Lhcb3 proteins; "pigment 2" SDF is a superposition of the on average second-lowest-energy pigment SDFs in adjacent monomers, etc. The three contributions to the HB action spectrum discussed in Section 3.1 and in the Supplemental Section are not the lowestenergy states of Lhcb1...3 proteins, respectively.)

Note that both Chl 604 and Chl 611 are absent in CP29 (See Table 2 where they are highlighted in bold). Figure 3 represents the structural arrangement of chlorophylls in LHCII. As the properties of the second-(or third-) lowest state of LHCII are significantly 
affected by monomerization (lower than lowest-state HB yield for the monomer, higher than lowest-state HB yield for the trimer), one could speculate that this state is localized on Chls 613 and 614 which are the closest to the interface between the monomers in the trimer. It is much less likely that this state is localized on Chls 602 and 603. The Chls 602 and 603 of the adjacent monomers are close to each other according to the trimer structure (Figure 3), and energy transfer between Chls 602 and 603 belonging to adjacent monomers would suppress burning into the second- (or third-) lowest state of the trimer with respect to the monomer. In reality, however, that state contributes more to the action spectrum of the trimer than to the action spectrum of the monomer.

The parameters of tunneling distribution may also be employed for disentangling the effects of this distribution and of the distribution of the EET rates in the hole burning data obtained for the higher-energy states. The first demonstration of fitting spectral holes using both tunneling parameter and B800 $\rightarrow$ B850 EET rate distributions for LH2 complex can be found in Ref. 38. Detailed discussion on retrieving EET rate distributions of various realistic shapes (e.g. obtained in purely Förster model, extended Förster models, etc) from the hole burning data is beyond the scope of this manuscript; separate manuscript devoted to this issue is in preparation. Briefly, distribution of EET rates has relatively small effect on the hole growth kinetics curves, providing some additional dispersion of HB yields in addition to that originating from the tunneling parameter distribution if the latter has parameters reported here, i.e. for $\sigma_{\lambda} \sim 1 \ldots 2$. The dependence of the hole width on hole depth, on the other hand, is fairly sensitive to the presence of the EET rate (homogeneous line width) distribution. 
Finally, we address the LHCII electron-phonon coupling parameters following from this work and their disagreement with $\Delta$-FLN data from ${ }^{19}$. Use of the parameters derived from $\Delta$-FLN data ${ }^{19}$ results in poor fits to the hole growth kinetics curves in case of both monomeric and trimeric LHCII, and in both cases Huang-Rhys factors had to be increased considerably to explain our HGK data. Obviously, this discrepancy can be attributed just to the differences between the species (pea in this work versus spinach in ${ }^{19}$ ). According to Liu et al. ${ }^{70}$, the sequence of LHCII from pea and spinach are only $89 \%$ identical, and some conserved residues are somewhat differently oriented. One may note, however, that both monomer and trimer data can be brought into agreement with the results of Ref. 19 if one assumes the presence of a very poorly-burning fraction contributing at least $15 \%$ of absorption in the low-energy region in our samples. This number did not depend on the degree of aggregation of the sample (partially aggregated samples were sometimes obtained by accident and explored in the course of this work, results not depicted). We also confirmed by modeling that reducing the lifetime of that fraction to $450 \mathrm{ps}$, the value expected at 683-684 $\mathrm{nm}$ for aggregated samples ${ }^{58}$ without any other modifications to model parameters is not sufficient to explain our HGK results. Note that the change of the lifetime alone affects only the burning rate, but not the maximal depth achievable. Consequently, poor burning of this fraction (if it is indeed present) is unlikely related to aggregation. Thus, increased electron-phonon coupling, either for a fraction of the complexes, or for all of them, is the most likely reason for smaller than expected maximal hole depth. We note here that an important difference between LHCII employed in this work and earlier ones is that LHCII explored here was from a fraction transiently associated with PS I, not obtained by regular procedures from 
PS II. Thus, our sample is expected to contain negligible amount of Lhcb3 protein ${ }^{71}$. The latter, normally comprising about 11\% of LHCII samples (6:2:1 Lhcb1:Lhcb2: Lhcb3; ${ }^{72}$ ) is known to exhibit the most red-shifted lowest state at room temperature. Samples explored in ${ }^{19}$ featured 4:4:2 ratio. Another expected consequence of our LHCII being associated with PS I rather than PS II is relative enrichment in Lhcb2 ${ }^{73,74}$, which is exhibiting the most blue-shifted lowest-energy state among the Lhcb1-3 proteins. It would be tempting to suggest that it is Lhcb2 which exhibits larger electron-phonon coupling. Attributing the effect to poor burning into one of the Lhcb proteins naturally explains the fact that same correction factor can be employed for both monomer and trimer: both were isolated in the same procedure from the same raw material, i.e., growth conditions were identical.

\section{Conclusions:}

Spectral hole growth kinetics measurements provide, along with SHB and SPCS spectral diffusion experiments, valuable information on the distributions of the barriers on the protein energy landscapes. Evolution of the spectral holes during burning depends on the distribution of the barriers in the excited electronic state of the pigment / protein system. (Experiments on broadening of spectral holes in the dark probe the ground state barrier distributions. SPCS, at liquid helium temperatures, provides information chiefly on the excited state; thermally-induced spectral diffusion is probed when the temperature is increased.) The distributions of the tunneling parameter $\lambda$ have been determined for the lowest-energy pigments of several photosynthetic pigment-protein complexes. It has been demonstrated that the hole burning yields are systematically significantly lower in 
photosynthetic proteins than in simple molecular glasses, in line with the idea that the barriers crossed by tunneling are higher and/or broader in protein; i.e. the groups of atoms whose light-induced rearrangement is manifesting via NPHB are larger. No evidence for fast $\sim 1 \mathrm{~cm}^{-1}$-shift spectral diffusion has been observed. While it has been known before that hole burning yields tend to be lower in proteins than in glasses, this study is the first where several pigment-protein complexes are addressed in a systematic and quantitative manner, similar to and easily comparable to that employed for glasses ${ }^{13,34-37}$. The barrier distribution parameters can be employed in spectral diffusion modeling potentially providing joint interpretation of light-induced spectral diffusion phenomena observed via SHB and SPCS, and as the benchmarks to which the results of future SPCS experiments could be compared to ensure that SPCS light-induced spectral diffusion data is in agreement with ensemble averages. The differences in protein dynamics parameters can be utilized to distinguish between pigments with strongly overlapping bands, along with the differences in electron-phonon coupling, pressure-induced shifts or permanent dipole moment change (see Ref. 13 for review). The parameters of tunneling distribution are also necessary for disentangling the effects of this distribution and of the distribution of EET rates in the hole burning data obtained for higher-energy states of PS complexes. Additionally, we attempted to assign the lowest-energy states of LHCII complex to particular chlorophylls known from structure data. The lowest-energy state of LHCII, in both trimeric and monomeric form, can be assigned either to Chl 604, strongly interacting only to Chls $b$, or to excitonically coupled Chls 610,611 and 612. Most likely different pigment(s) are responsible for the lowest state of the CP29, as barrier distribution parameters of CP29 differ significantly from those of LHCII. 
Acknowledgments: Research at Concordia University is supported by NSERC and CFI. We thank our collaborator Dr. Nathan Nelson (Tel Aviv University) for helping with LHCII purification, and Dr. Tõnu Reinot (ISU) for useful discussions on HGK modeling. Xin Zhao is acknowledged for buffer preparation. R.P. would like to thank Spanish MICINN (Grant AGL2008-00377). M. S. acknowledges the contribution of Energy Biosciences Program, Basic Energy Sciences, USDOE. J. P. and K.-D. I. gratefully acknowledge support from Deutsche Forschungsgemeinschaft (SFB 429, TP A1 and TP A3, respectively). 
Table 1: HGK curves simulation parameters. Fit parameters correspond to the lowest burn frequencies which are highlighted in bold.

\begin{tabular}{|c|c|c|c|c|}
\hline Complex & CP43, A-state & СР29 & LHCII monomer & LHCII trimer \\
\hline $\begin{array}{l}\text { “pigment 1” SDF } \\
\text { peak and width } \\
\left(\mathrm{cm}^{-1}\right)\end{array}$ & $14641 ; 180$ & $14734,170^{a}$ & $14705 ; 200^{\mathrm{b}}$ & $14738,200^{c}$ \\
\hline $\begin{array}{l}\text { Oscillator strength } \\
\text { (Chl } a \text { equivalents) }\end{array}$ & 1.0 & 1.0 & 1.2 & 1.2 \\
\hline Lifetime $\tau_{1}$ (ns) & 3.5 & 4.8 & 4.4 & 4.4 \\
\hline$\Gamma_{\text {homog }}\left(\mathbf{c m}^{-1}\right)$ & 0.03 & 0.04 & 0.04 & 0.04 \\
\hline Burn frequency & 14560 & 14670 & 14616 & 14640 \\
\hline \multirow[t]{2}{*}{$\omega_{\mathrm{B}}\left(\mathrm{cm}^{-1}\right)$} & & 14694 & 14628 & 14652 \\
\hline & & 14720 & 14653 & 14656 \\
\hline SPSB & $0.30 \pm 0.05$ & $0.65 \pm 0.05$ & $0.80 \pm 0.05^{d}$ & $1.3 \pm 0.1^{\mathrm{c}}$ \\
\hline $\begin{array}{c}\text { PSB: } \omega_{\mathrm{m}} ; \Gamma_{\text {Gauss }} ; \\
\Gamma_{\text {Lorentz }}\left(\mathrm{cm}^{-1}\right)\end{array}$ & $17 ; 15 ; 70$ & $22 ; 20 ; 110$ & $22 ; 20 ; 110^{d}$ & $22 ; 20 ; 110$ \\
\hline$\lambda_{0}$ & $11.0 \pm 0.2$ & $10.2 \pm 0.2$ & $11.3 \pm 0.4^{\mathrm{d}}$ & $11.2 \pm 0.4^{\mathrm{d}}$ \\
\hline$\sigma_{\lambda}$ & $1.0 \pm 0.05$ & $1.4 \pm 0.2$ & $2.0 \pm 0.4$ & $2.3 \pm 0.4$ \\
\hline
\end{tabular}

${ }^{\mathrm{a}}$ SDF parameters from ${ }^{17}$ : peak $14745 \mathrm{~cm}^{-1}$, FWHM $=120 \mathrm{~cm}^{-1}$, S=0.4-0.6.

${ }^{\mathrm{b}}$ SDF parameters from ${ }^{19}$ : peak $14715 \mathrm{~cm}^{-1}$, FWHM $=110 \mathrm{~cm}^{-1}, \mathrm{~S}=0.6$

${ }^{\text {c }}$ SDF parameters from ${ }^{19}$ : peak $14705 \mathrm{~cm}^{-1}$, FWHM $=80 \mathrm{~cm}^{-1}$; $\mathrm{S}=0.8-0.9$. In our model $\sim 80-100 \mathrm{~cm}^{-1}$ width is attributed to the SDF of the second-lowest energy pigment.

${ }^{\mathrm{a}}$ The best fit with electron-phonon coupling parameters exactly as in ${ }^{19}$ yields $\lambda_{0}=12.2$ 
Table 2. Chlorophyll labeling for LHCII and CP29

\begin{tabular}{|c|c|c|c|c|}
\hline $\begin{array}{l}\text { Standfuss } \\
\text { et al }\end{array}$ & Liu et al ${ }^{70}$ & $\begin{array}{l}\text { Kühlbrandt } \\
\text { et al }^{76}\end{array}$ & $\begin{array}{c}\text { CP29 (modeling usually } \\
\text { based on Kühlbrandt et al) }\end{array}$ & Chl $a$ or $b$ \\
\hline 1 & 610 & A1 & A1 & A \\
\hline 2 & 612 & A2 & A2 & A \\
\hline 3 & 613 & A3 & $A 3^{\mathrm{a}}$ & A \\
\hline 4 & 602 & A4 & A4 & A \\
\hline 5 & 603 & A5 & A5 & A \\
\hline 6 & 604 & A6 & & A \\
\hline 7 & 611 & B2 & & A \\
\hline 8 & 614 & B3 & $\mathrm{B}^{\mathrm{a}}$ & A \\
\hline 9 & 601 & & & B \\
\hline 10 & 607 & A7 & & B \\
\hline 11 & 608 & B1 & & B \\
\hline 12 & 609 & B5 & $B 5^{\mathrm{a}}$ & B \\
\hline 13 & 606 & B6 & $B 6^{\mathrm{a}}$ & $\mathrm{B}$ \\
\hline 14 & 605 & & & B \\
\hline
\end{tabular}

${ }^{\mathrm{a}}$ mixed sites in CP29 according to mutagenesis studies by Bassi et al ${ }^{77}$ 


\section{References:}

1. Hayes, J. M., Small, G. J., J. Phys. Chem. 1986, 90, 4928.

2. Gillie, J. K., Small, G. J., Golbeck, J. H., J. Phys. Chem. 1989, 93, 1620.

3. Groot, M. L., Dekker, J. P., van Grondelle, R., den Hartog, F. T. H., Völker, S., J. Phys. Chem., 1996, 100, 11488.

4. Wu, H.-M.; Rätsep, M., Lee, I.-J.; Cogdell, R. J.; Small, G. J., J. Phys. Chem B 1997, 101, 7654 .

5. den Hartog, F. T. H.; Dekker, J. P.; van Grondelle, R.; Völker, S. J. Phys. Chem. B 1998, 102, 11007.

6. Creemers, T. M. H.; den Hartog, F. T. H.; Störkel, U.; Völker, S. in Photosynthesis: Mechanisms and Effects, Vol 1, G. Garab, ed., Kluwer, 1998, pp. 445-448.

7. Störkel, U.; Creemers, T. M. H.; den Hartog, F. T. H.; Völker, S., J. Lumin. 1998, 76-77, 327.

8. den Hartog, F. T. H.; Vacha, F., Lock, A. J., Barber, J., Dekker, J. P. Völker, S., J. Phys. Chem. B 1998, 102, 9174.

9. den Hartog, F. T. H.; van Papendrecht, C.; Störkel, U.; Völker, S. J. Phys. Chem. B 1999, 103, 1375.

10. Pieper, J., Rätsep, M., Jankowiak, R., Irrgang, K.-D., Voigt, J., Renger, G., Small, G. J., J. Phys. Chem. A 1999, 103, 2412.

11. Pieper, J., Irrgang, K.-D., Rätsep, M., Voigt, J., Renger, G., Small, G. J., Photochem. Photobiol. 2000, 71, 574.

12. Jankowiak, R., Zazubovich, V., Rätsep, M., Matsuzaki, S., Alfonso, M., Picorel, R., Seibert, M., Small, G. J., J. Phys. Chem. B 2000, 104, 11805. 
13. Reinot, T., Zazubovich, V., Hayes, J. M., Small, G. J., J. Phys. Chem. B 2001, 105, 5083.

14. Hughes, J. L., Prince, B. J., Peterson Ärsköld, S., Krausz, E., Pace, R. J., Picorel, R., Seibert, M., J. Lumin. 2004, 108, 131.

15. Riley, K. J., Jankowiak, R., Rätsep, M., Small, G. J., Zazubovich, V., J. Phys. Chem. B 2004, 108, 10346.

16. Hughes, J. L., Picorel, R., Seibert, M., Krausz, E., Biochemistry 2006, 45, 12345.

17. Rätsep, M., Pieper, J., Irrgang, K.-D., Freiberg, A., J. Phys. Chem. B 2008, 112, 110.

18. Reppert, M., Zazubovich, V., Dang, N. C., Seibert M., Jankowiak, R., J. Phys. Chem. B 2008, 112, 9921.

19. Pieper, J., Rätsep, M., Irrgang, K.-D., Freiberg, A., J. Phys. Chem. B 2009, 113, 10870.

20. Gibasiewicz, K., Rutkowski, M., van Grondelle, R., Photosynthetica 2009, 47, 232.

21. Purchase, R., Völker, S., Photosynth. Res. 2009, 101, 245.

22. Hofmann, C., Aartsma, T. J., Michel H., Köhler, J., Proc. Natl. Acad. Sci. USA 2003, 100, 15534.

23. Baier, J., Richter, M. F., Cogdell, R. J., Oellerich, S., Köhler, J. J. Phys. Chem. B 2007, 111, 1135.

24. Oikawa, H., Fujiyoshi, S., Dewa, T., Nango, M., Matsushita M., J. Am. Chem. Soc. 2008, 130, 4580 .

25. Baier, J., Richter, M. F., Cogdell, R. J., Oellerich, S., Köhler, J. Phys. Rev. Lett. 2008, 100, 018108. 
26. Baier, J., Gabrielsen, M., Oellerich, S., Michel, H., van Heel, M., Cogdell, R. J., Köhler, J., Biophys. J. 2009, 97, 2604.

27. Berlin, Y., Burin, A., Friedrich, J., Köhler, J., Phys. Life Rev. 2007, 4, 64.

28. Berlin, Y., Burin, A., Friedrich, J., Köhler, J., Phys. Life Rev. 2006, 3, 262.

29. Leeson D. T., Wiersma, D. A., Fritsch, K., Friedrich, J., J. Phys. Chem. B, 1997, 101 6331

30. Ponkratov, V. V., Friedrich, J., Markovic, D., Scheer, H., Vanderkooi, J., M., J. Phys. Chem. B, 2004, 108, 1109.

31. Vainer, Yu. G., Naumov, A. V., Bauer M., Kador, L., J. Lumin. 2004, 107, 287.

32. Naumov, A. V., Vainer, Yu. G., Bauer M., Kador, L., J. Chem. Phys. 2003, 119, 6296.

33. Zilker, S. J., Kador, L., Friebel, J., Vainer, Yu. G.,, Kol'chenko, M. A., Personov, R. I., J. Chem. Phys. 1998, 109, 6780.

34. Reinot, T., Dang, N. C., Small, G. J., J. Lumin. 2002, 98, 183.

35. Reinot, T., Small, G. J., J. Chem. Phys. 2001, 114, 9105.

36. Reinot, T., Dang, N. C., Small, G. J., J. Chem. Phys. 2003, 119, 10404.

37. Dang, N. C., Reinot, T., Reppert, M., Jankowiak, R., J. Phys. Chem. B 2007, 111, 1582.

38. Grozdanov, D., Herascu, N., Reinot, T., Jankowiak, R., Zazubovich, V., J. Phys. Chem. B., 2010, 114, 3426.

39. Riley, K. J., Reinot, T., Fromme, P., Jankowiak, R., Zazubovich, V., J. Phys. Chem. B 2007, 111, 286.

40. Anderson, P. W., Halperin, B. I., Varma, C. M., Phil. Mag., 1972, 25, 1. 
41. Phillips, W. A., J. Low. Temp. Phys. 1972, 7, 351

42. Jankowiak, R., Small, G. J., Science 1987, 237, 618.

43. Shu, L., Small, G. J., J. Opt. Soc. Am. B 1992, 9, 724.

44. Frauenfelder, H., Sligar, S. G., Wolynes, P.G., Science 1991, 254, 1598.

45. Fenimore, P. W., Frauenfelder, H., McMahon, B. H., Young, R. D., Physica A 2005, 351,1

46. Janusonis, J., Valkunas, L., Rutkauskas, D., van Grondelle, R., Biophys. J. 2008, 94, 1348.

47. Rutkauskas, D., Novoderezhkin, V. I., Cogdell, R. J., van Grondelle, R., Biophys. J. 2005, 88, 422.

48. Novoderezhkin, V. I., Rutkauskas, D., van Grondelle, R., Biophys. J. 2006, 90, 2890.

49. Amunts, A., Ben-Shem, A. Nelson, N., Photochem Photobiol. Sci. 2005, 4, 1011.

50. Jelezko, F., Tietz, C., Thews, E., Schuler, S., Wechsler, A, Wrachtrup, J., Optics and Spectroscopy, 2001, 91, 486

51. Tietz, C., Jelezko, F., Gerken, U., Schuler, S., Schubert, A., Rogl, H., Wrachtrup, J., Biophys. J., 2001, 81, 556.

52. Zazubovich, V., Jankowiak, R., J. Lumin. 2007, 127, 245.

53. Peterman, E. J. G., Pullerits, T., van Grondelle, R., van Amerongen, H., J. Phys. Chem. B 1997, 101, 4448.

54. Reppert, M., Zazubovich, V., Dang, N. C., Seibert M., Jankowiak, R., J. Phys. Chem. B 2008, 112, 9934. 
55. Loll, B., Kern, J., Zouni, A., Saenger, W., Biesiadka, J., Irrgang, K.-D., Photosynth. Res. 2005, 86, 175.

56. Huyer, J., Eckert, H.-J., Irrgang, K.-D., Miao, J., Eichler H.-J., Renger, G., J. Phys. Chem. B 2004, 108, 3326.

57. Pieper, J., Irrgang, K.-D., Rätsep, M., Jankowiak, R., Schrötter, T., Voigt, J., Small, G. J., Renger, G., J. Phys. Chem. A 1999, 103, 2422.

58. Vasil'ev, S., Irrgang, K.-D., Schrötter, T., Eichler, H.-J., Renger, G., Biochemistry. 1997, 36, 7503.

59. Reddy, N. R. S., van Amerongen, H., Kwa, S. L. S., van Grondelle, R., Small, G. J., J. Phys. Chem. 1994, 98, 4729.

60. Tietz, C., Gerken, U., Jelezko, F., Wrachtrup, J., Single Mol. 2000, 1, 67.

61. Gerken, U., Wolf-Klein, H., Huschenbett, C., Götze, B., Schuler, S., Jelezko, F., Tietz, C., Wrachtrup, J., Paulsen, H., Single Mol. 2002, 4, 183.

62. Palacios, M. A., de Weerd, F. L., Ihalainen, J., A., van Grondelle, R., van Amerongen, H., J. Phys. Chem. B 2002, 106, 5782.

63. Du, H., Fuh, R. A., Li, J., Corkan, A., Lindsey, J. S., Photochem. Photobiol., 1998, 68, 141.

64. Di Donato, M., van Grondelle, R., van Stokkum, I. H. M., Groot, M. L., J. Phys. Chem. B 2007, 111, 7345.

65. He, J.-F., Wang S.-C., Zhang S., He, F.-T., Shan, J.-X., Kuang, T.-Y., Acta Botanica Sinica / Journal of Integrative Plant Biology 2001, 43, 704.

66. Krüger, T. P. J., Novoderezhkin, V. I., Ilioaia, C., van Grondelle, R., Biophys. J., 2010, 98, 3093. 
67. Novoderezhkin, V. I., Palacios, M. A., van Amerongen, H., van Grondelle, R., J. Phys. Chem. B 2005, 109, 10493.

68. Linnanto, J., Martiskainen, J., Lehtovuori, V., Ihalainen, J., Kananavicius, R., Barbato, R., Korppi-Tommola, J., Photosynth. Res. 2006, 87, 267

69. Shlau-Cohen, G., Calhoun, T. R., Ginsberg, N. S., Read, E. L., Ballottari, M., Bassi, R., van Grondelle, R., Fleming, G. R., J. Phys. Chem. B 2009, 113, 15352.

70. Liu, Z., Yan, H., Kuang, T., Zhang, J., An, X., Chang, W., Nature, 2004, 428, 287.

71. Standfuss, J., Kühlbrandt, W., J. Biol. Chem., 2004, 279, 36884.

72. Jackowski, G., Kacprzak, K., Jansson, S., Biochim. Biophys. Acta 2001, 1504, 340.

73. Larsson, U. K., Andersson, B., Biochim. Biophys. Acta 1985, 809, 396.

74. Larsson, U. K., Sundby, C., Andersson, B., Biochim. Biophys. Acta 1987, 894, 59.

75. Standfuss, J.; Lamborghini, M.; Kühlbrandt, W.; van Scheltinga, A. C. T., EMBO J. 2005, 24, 919.

76. Kühlbrandt, W.; Wang, D. N.; Fujiyoshi, Y. Nature 1994, 367, 614.

77. Bassi, R., Croce, R., Cugini, D., Sandona, D., Proc. Natl. Acad. Sci. USA 1999, 96, 10056. 


\section{Figure Captions:}

Figure 1. $5 \mathrm{~K}$ absorption (red curves) and low-dose HB action spectra (symbols) of CP43 (A), CP29 (B), trimeric LHCII (C) and monomeric LHCII (D). Arrows indicate burn wavelengths. In case of CP29 (B) two action spectra are reported: black diamonds, for irradiation dose of $0.0002 \mathrm{~J} / \mathrm{cm}^{2}$ and green triangles, for $0.0014 \mathrm{~J} / \mathrm{cm}^{2}$. Blue curves represent the $5 \mathrm{~K}$ fluorescence spectra obtained with excitation at $650 \mathrm{~nm}$. Dashed black curves in frames B-D are Gaussian fits to the HB action spectra.

Figure 2. A: The HGK curve (noisy black curve) and its fit (red) for CP43 at $686.8 \mathrm{~nm}$. The insert contains the high-resolution scan of the hole burned during the HGK measurement reported in the main frame. B: The HGK curve (noisy black curve) and its fit (red) for CP29 at $681.7 \mathrm{~nm}$. C: The HGK curve (noisy black curve) and its fit (red) for trimeric LHCII at $683.1 \mathrm{~nm}$. D: The HGK curve (noisy black curve) and its fit (red) for monomeric LHCII at $684.2 \mathrm{~nm}$. The insert contains the high-resolution scan of a 15\% hole burned at $\sim 684 \mathrm{~nm}$.

Figure 3. Arrangement of chlorophyll molecules in the LHCII trimer. View from the stromal side. LHCII protein backbone is shown as semitransparent, chlorophylls are shown in sticks with Mg as a yellow sphere. Chlorophylls 610, 611 and 612 are red; chlorophylls 613 and 614 are blue, chlorophylls 602 and 603 are green and Chl 604 is in yellow. Chl 604 is located at the luminal side of the complex. The rest of the chlorophylls are shown in transparency. Dashed ellipse approximately delineates the borders of one monomer. 